PROCEEDINGS OF THE

AMERICAN MATHEMATICAL SOCIETY

Volume 126, Number 4, April 1998, Pages 957-964

S 0002-9939(98)04146-X

\title{
INCOHERENT NEGATIVELY CURVED GROUPS
}

\author{
DANIEL T. WISE \\ (Communicated by Ronald M. Solomon)
}

\begin{abstract}
In part 1, a construction of Rips is modified so that it produces a CAT $(-1)$ group instead of a small-cancellation group. Thus, many of the applications of Rips' construction to small-cancellation groups may be applied to CAT $(-1)$ groups as well. Part 2 offers a simple way of producing incoherent groups.
\end{abstract}

\section{INTRODUCTION}

Given a finitely presented group $Q$, a construction of Rips' [10] provides a short exact sequence

$$
1 \rightarrow N \rightarrow G \rightarrow Q \rightarrow 1
$$

where $G$ is a finitely presented small-cancellation group and $N$ is a finitely generated normal subgroup, generated by two elements of $G$.

Applications of Rips' construction generally involve lifting some property (usually a 'subgroup pathology') from an arbitrary (usually finitely presented) group $Q$ to the small-cancellation group $G$. For instance, in [10] Rips uses his construction to show that there are examples of small-cancellation groups that do not have the finitely generated intersection property. In [3], Rips' construction is used to show that various problems about small-cancellation groups are recursively unsolvable. In [2], Rips' construction is used to find Automatic groups with non-Automatic finitely-presented subgroups.

A group is said to be coherent [12] if every finitely generated subgroup is finitely presented. The question of whether or not $C(8)$ small-cancellation groups are coherent was resolved negatively by Rips using his construction [10]. The main objective of this paper is to answer a question of Gersten's [7], who asked if fundamental groups of negatively curved 2-complexes are coherent. Parts 1 and 2 of this paper provide methods for constructing incoherent negatively curved groups.

In part 1 of this paper, I provide a construction similar to that of Rips, but in which $G$ is the fundamental group of a compact negatively curved 2-complex instead of a small-cancellation group.

1.3 Theorem: Adapted Rips construction. Let $Q$ be a finitely presented group. Then there exists a group $G$ which is the fundamental group of a compact negatively curved 2-complex and a finitely generated normal subgroup $N \triangleleft G$ such that $G / N \cong Q$.

Received by the editors March 17, 1996.

1991 Mathematics Subject Classification. Primary 20F32, 20 F05.

(C)1998 American Mathematical Society 
It should be mentioned that the 2-complexes corresponding to Rips' construction do not admit metrics of negative curvature. This construction also differs from that of Rips' in the number of generators of $N$. While Rips' normal subgroup $N$ is generated by only 2 elements of $G$, the normal subgroup $N$ of this paper is generated by many elements of $G$, and the number of such generators grows with the length of the presentation for $Q$. Allowing $N$ to be generated by many elements instead of just 2, makes it easier to accomodate the somewhat stricter negative curvature condition.

Thus, the applications of Rips' construction in [2], [3], and [10] mentioned above may be extended to finding examples of fundamental groups of compact negatively curved 2-complexes (or where appropriate, non-positively curved complexes) exhibiting the various pathologies. For example, Corollary 1.5 provides certain pathological examples of negatively curved groups analogous to the pathological examples of small-cancellation groups given in [10]. In particular, it is shown how to construct an incoherent negatively curved group.

In part 2 of this paper, I provide a different method, which is more geometric, for constructing incoherent finitely presented groups (see Theorem 2.1). The essential idea is to build a group in such a way that it contains a finitely generated subgroup with (an easily determined) infinitely generated $H_{2}$. This method is somewhat more transparent than that of using Rips' construction and I hope that it sheds some more light on incoherent groups.

Using this method, I provide an easy example of a compact negatively curved 2-complex with incoherent fundamental group. Note, that while the method of part 1 yields a plethora of such examples, they are all quite complicated.

It is worth mentioning that it is easy to give examples of compact non-positively curved 2-complexes with incoherent fundamental group. Indeed, $F_{2} \times F_{2}$, the product of two free groups of rank 2 , is incoherent, and is the fundamental group of a non-positively curved squared complex. See also [4].

The class of coherent groups is not very well understood. It includes certain small groups, like finite groups and nilpotent groups. Scott [11] proved that it includes all 3-manifold groups. Serre asks whether $S L_{3}(Z)$ is coherent. It is also asked [1] whether 1-relator groups are coherent. Note that Example 2.3 is a 2-relator group which is incoherent.

\section{Part 1: AdAPted Rips' CONSTRUCtion}

1.1 Definition: No 2-letter repetitions. A set of words $V_{1}, \ldots, V_{v}$ in some alphabet $B=\left\{b_{1}, \ldots, b_{n}\right\}$ is said to have no 2-letter repetitions if each 2-letter word in $B$ appears as a subword of at most one of the $V_{i}$, and in at most one place in each of the $V_{i}$. (In the language of small-cancellation theory, this is nearly equivalent to no pieces of length $\geq 2$.)

1.2 Lemma: Long word with no 2-letter repetitions. Let $J$ be a positive integer, and let

$$
X_{J}=\left\{x_{1}, x_{2}, \ldots, x_{J}\right\}
$$

be a set of $J$ letters. Then there is a (positive) word $\Sigma_{J}$ in $X_{J}$ of length $J^{2}$ which has the property that no 2-letter word in $X_{J}$ appears more than once in $\Sigma_{J}$ as a subword. 
Proof. Consider the sequence

$$
\begin{aligned}
& \Sigma_{1}=\left(x_{1}\right) \\
& \Sigma_{2}=\left(x_{1} x_{1} x_{2}\right)\left(x_{2}\right) \\
& \Sigma_{3}=\left(x_{1} x_{1} x_{2} x_{1} x_{3}\right)\left(x_{2} x_{2} x_{3}\right)\left(x_{3}\right) \\
& \Sigma_{4}=\left(x_{1} x_{1} x_{2} x_{1} x_{3} x_{1} x_{4}\right)\left(x_{2} x_{2} x_{3} x_{2} x_{4}\right)\left(x_{3} x_{3} x_{4}\right)\left(x_{4}\right) .
\end{aligned}
$$

In general, define $\Sigma_{J}$ to be the following word:

$$
\Sigma_{J}=\left(x_{1} x_{1} x_{2} x_{1} x_{3} x_{1} x_{4} \cdots x_{1} x_{J}\right)\left(x_{2} x_{2} x_{3} x_{2} x_{4} \cdots x_{2} x_{J}\right) \cdots\left(x_{J-1} x_{J-1} x_{J}\right)\left(x_{J}\right) .
$$

It is easy to see that

$$
\left\|\Sigma_{J}\right\|=\sum_{i=1}^{J}(2 i-1)=J^{2} .
$$

Furthermore, it is easy to see that $\Sigma_{J}$ has no repetitions of a 2-letter subword.

1.3 Theorem: Adapted Rips construction. Let $Q$ be a finitely presented group. Then there exists a group $G$ which is the fundamental group of a compact negatively curved 2-complex and a finitely generated normal subgroup $N \triangleleft G$ such that $G / N \cong Q$.

Proof. Let $Q$ be presented by

$$
\left\langle q_{1}, \ldots, q_{I} \mid R_{1}, \ldots, R_{K}\right\rangle .
$$

We will assume that each of the relators is a reduced word.

Following Rips' construction, we wish to choose a presentation for $G$ of the form

$$
\left\langle\begin{array}{c|c}
q_{1}, \ldots, q_{I} & x_{j}^{q_{i}}=W_{i j+}, \\
x_{1}, \ldots, x_{J} & x_{j}^{q_{i}^{-1}}=W_{i j-},
\end{array} R_{k}=W_{k}\right\rangle \quad\left(\begin{array}{l}
i \in\{1, \ldots, I\} \\
j \in\{1, \ldots, J\} \\
k \in\{1, \ldots, K\}
\end{array}\right)
$$

where $W_{i j \pm}$ and $W_{k}$ are positive words of length 14 and $2\left\|R_{k}\right\|+8$, respectively, in the $X_{J}$ letters, as will be described below. Also, we will postpone our choice of $J$ and thus the number of additional generators, until after it is motivated below. Note that we use the notation $b^{a}=a b a^{-1}$ to denote conjugation by $a$. One sees immediately that the finitely generated subgroup $N=\left\langle X_{J}\right\rangle$ is a normal subgroup of $G$, and furthermore $G / N \cong Q$.

We wish to show that with the appropriate choices, the standard 2-complex of the above presentation for $G$ will have a metric of negative curvature. In order to motivate the choices of $W_{i j \pm}$ and $W_{k}$ we consider the diagrams of Figure 1 . We have divided the polygons in Figure 1, corresponding to the relations of the presentation of $G$, into pentagons.

If we were to metrize each of these pentagons as a regular hyperbolic pentagon with angle $\frac{\pi}{2}$, then any corner of our polygons containing a $q$-edge would have angle $\pi$. This greatly simplifies what must be verified in order for the link condition (see [6], [8] ) to be satisfied. Since we have assumed above that the words $R_{i}$ are reduced, there is no corner with label $q_{i} q_{i}^{-1}$. Therefore any cycle of corners containing some black (i.e., $q$-labelled) edge must have total angle sum $\geq 2 \pi$. Thus it is sufficient to consider sequences of corners not containing any black edges. 

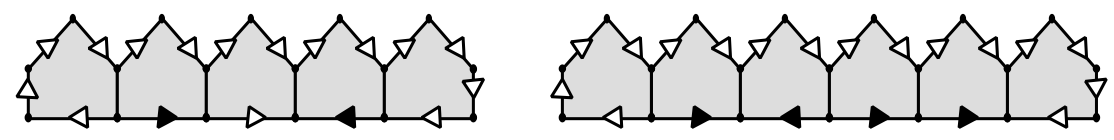

Figure 1. The polygon on the left corresponds to a relation of type $x_{j}^{q_{i}}=W_{i j+}$. It is formed by gluing together 5 regular hyperbolic pentagons with angle $\frac{\pi}{2}$. The polygon on the right corresponds to a relation of type $R_{k}=W_{k}$. It is formed by gluing together $\left\|R_{k}\right\|+2$ regular hyperbolic pentagons with angle $\frac{\pi}{2}$. In both figures, the black arrows label $q$ edges, and the white arrows label $x$ edges. The long words $W_{i j \pm}$ and $W_{k}$ correspond to the long sequence of white edges.

Because we chose the words $W_{i j \pm}$ and $W_{k}$ to be positive, the all-white (i.e., containing only $x$-labelled edges) corners have labels which are of the form $x_{j} x_{h}$ (or of the form $x_{h}^{-1} x_{j}^{-1}$ ). It is easy to see that any all-white cycle must have even length. However, since any corner has angle $\geq \frac{\pi}{2}$, it is sufficient to demand that there be no such cycles of length 2 , that is, no two corners have the same $x_{j} x_{h}$ label. In other words, we need only choose the set of words $\left\{W_{i j+}, W_{i j-}, W_{k} \mid 1 \leq\right.$ $i \leq I, 1 \leq j \leq J, 1 \leq k \leq K\}$ so that it has no 2-letter repetitions.

Finally, we choose $J$ to be an integer large enough so that $J^{2}$ exceeds the total length of the $2 I J$ distinct $W_{i j \pm}$ words of length 14 , and the $K$ distinct $W_{k}$ words each of length $2\left\|R_{k}\right\|+8$. So

$$
J^{2} \geq(2 I J) 14+\sum_{k=1}^{K}\left(2\left\|R_{k}\right\|+8\right) .
$$

It now follows from the lemma that there is a (positive) word $\Sigma_{J}$ in $\left\{x_{1}, \ldots, x_{J}\right\}$ whose length is $J^{2}$ and which has no 2-letter repetitions. Because of equation (1), we see that $\Sigma_{J}$ is long enough so that $\Sigma_{J}$ can be chopped up into a set of words $\left\{W_{1}, \ldots, W_{k}, W_{11+}, W_{11-}, \ldots, W_{I J+}, W_{I J-}\right\}$ which again has no 2-letter repetitions.

1.4 Remark. One is tempted to subdivide the polygons of the complex constructed above into pentagons as suggested by the diagrams. The derived complex obviously satisfies the small cancellation conditions $C(5)-T(4)$. In fact, it is not difficult to show, that for any large $n$ and $m$, one can produce a group $G$, designed to fit into the exact sequence above, so that $G$ is $\pi_{1}$ of a negatively curved 2-complex formed from regular hyperbolic $n$-gons with angle $\frac{2 \pi}{m}$. To do this, one chooses $\Sigma_{J}$ to satisfy the $T(m)$ condition, and one subdivides the polygons of Figure 1 into $n$-gons in a slightly more complicated fashion - making sure that there are at least $m n$-gons meeting at each vertex incident with a black edge.

Following Rips, we have the following corollary of Theorem 1.3:

1.5 Corollary. For suitable $Q$, we obtain a group $G$ which is the fundamental group of a negatively curved 2-complex such that:

(1) There are finitely generated subgroups $H_{1}$ and $H_{2}$ of $G$ such that $H_{1} \cap H_{2}$ is not finitely generated.

(2) $G$ is incoherent.

(3) The generalized word problem in $G$ is not solvable. 
Proof. We repeat the proofs given in [10]. Let $\phi$ denote the quotient map $G \rightarrow Q$ of Theorem 1.3. Note that if $K=\left\langle k_{1}, \ldots, k_{s}\right\rangle$ is a finitely generated subgroup of $Q$, then its preimage in $G, H=\phi^{-1}(K)$, is also finitely generated. Indeed, $H=\left\langle h_{1}, \ldots, h_{s}, x_{1}, \ldots, x_{J}\right\rangle$ where for each $i, \phi\left(h_{i}\right)=k_{i}$.

(1) Let $Q$ be a finitely presented group with a pair of finitely generated subgroups $K_{1}$ and $K_{2}$ whose intersection $K_{1} \cap K_{2}$ is not finitely generated. Let $H_{1}=\phi^{-1}\left(K_{1}\right)$ and $H_{2}=\phi^{-1}\left(K_{2}\right)$. Then $H_{1}$ and $H_{2}$ are finitely generated subgroups of $G$, but $H_{1} \cap H_{2}$ is infinitely generated because $\phi\left(H_{1} \cap H_{2}\right)=$ $K_{1} \cap K_{2}$.

(2) Let $Q$ be a finitely presented incoherent group; then $G$ is incoherent. This is because if $K \subset Q$ is a finitely generated subgroup which is not finitely presented, then $H=\phi^{-1}(K)$ is finitely generated but not finitely presented because $H / N \cong K$.

(3) Let $Q$ be a finitely presented group with unsolvable word problem; then the generalized word problem for $G$ with respect to $N$ is not solvable. This is because an algorithm which decides whether or not an element of $G$ is an element of $N$ could be used to decide if an element of $Q$ is trivial.

1.6 Remark. There is another way of using Rips' construction (or the adapted Rips construction of Theorem 1.3) in order to produce incoherent groups. A theorem of Bieri [5] states that a finitely presented normal subgroup of a finitely presented group of cohomological dimension 2 is either free or of finite index. Since (torsionfree) small-cancellation groups (or negatively curved 2-complexes respectively) are of cohomological dimension 2 [9], one may apply Bieri's theorem to the group $G$. It follows that if $Q$ is infinite, then either $N$ is free or $N$ is not finitely presented and so $G$ is incoherent.

In the case of Rips' construction, $N$ is generated by exactly 2 generators so any (non-trivial) relation shows that $N$ is not free, and such a relation is supplied easily. In the case of the adapted Rips construction of Theorem 1.3, it is not immediately obvious that in general $N$ is not free, though this is easy to check in special cases.

\section{PART 2: A RECIPE FOR INCOHERENT FINITELY-PRESENTED GROUPS}

Consider the finite presentation

$$
\left\langle a_{1}, \ldots, a_{m}, t \quad \mid R=1, \quad a_{1}^{t}=W_{1}, \ldots, a_{m}^{t}=W_{m}\right\rangle
$$

where $W_{i}$ and $R$ are words in $a_{1}^{ \pm 1} \ldots a_{m}^{ \pm 1}$. Let $X$ denote the standard 2-complex associated with this presentation. Let $G=\pi_{1}(X)$ and let $A \subset G$ denote the subgroup generated by $\left\{a_{1} \ldots a_{m}\right\}$.

2.1 Theorem: Aspherical $\Rightarrow$ Incoherent. If $X$ is aspherical and $R$ is not the empty word, then $\operatorname{rank}\left(H_{2}(A)\right)=\infty$ and so $A$ is not finitely presented.

Proof. Let $\hat{X}$ denote the cover of $X$ corresponding to the subgroup $A \subset G$. Let $B \subset X$ denote the subcomplex consisting of the 0-cell and the $a_{j}$ labelled 1-cells. Let $\hat{B} \subset \hat{X}$ denote the lift of $B$ at the basepoint.

As $X$ is a $\mathrm{K}(\pi, 1)$ space for $G, \hat{X}$ is a $\mathrm{K}(\pi, 1)$ space for $A$, so $H_{2}(A)=H_{2}(\hat{X})$.

Denote by $r$ the 2-cell of $X$ associated with the relation $R=1$. 

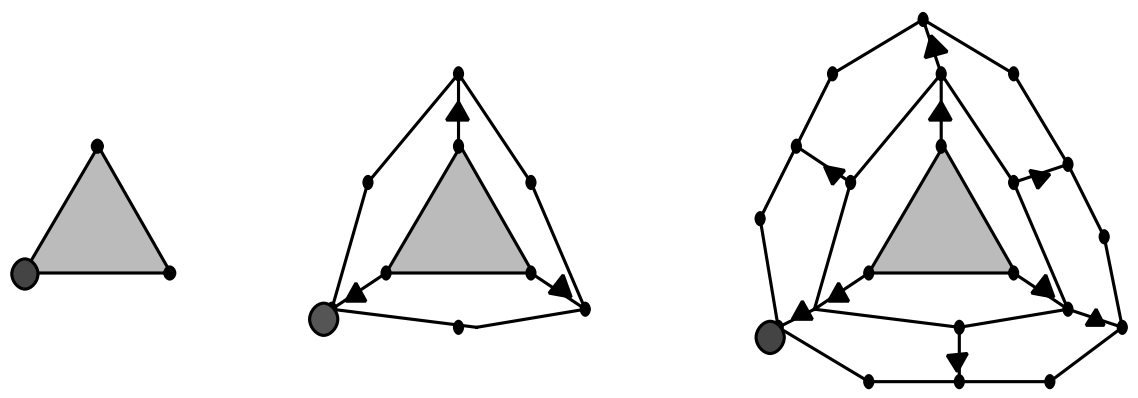

Figure 2. The sequence of diagrams in the above figure are meant to suggest $D_{0}, D_{1}$ and $D_{2}$ from left to right. The shaded triangle in the center of each of the diagrams corresponds to an $r$ 2-cell of $X$. The remaining 2-cells correspond to relations of the form: $a_{i}^{t}=W_{i}$. The edges with a black arrow corrrespond to $t$ letters. The basepoint of each diagram is the large grey vertex at its lowerleft corner.

For each integer $n \geq 0$ we will form a combinatorial disc-diagram $D_{n}$ mapped into $X$. Each disc-diagram in our sequence will have the property that $\partial D_{n}$ is mapped into $B$.

This sequence of disc-diagrams corresponds to a sequence of relations among the generators of $A$. The idea of the proof is to use the sequence of 2-chains corresponding to the lifts of the $D_{n}$ to show that $H_{2}(\hat{X})$ has infinite rank.

$D_{0}$ is the obvious disc-diagram corresponding to the 2-cell $r$. For each $n, D_{n+1}$ is an extension of $D_{n}$ by an annulus which is mapped into $X-r$. This annulus has width one and is composed of 2-cells corresponding to the $a_{i}^{t}=W_{i}$ relations joined along $t$-edges.

To be more explicit, let $M$ denote the free monoid on $\left\{a_{1}^{ \pm 1}, \ldots, a_{n}^{ \pm 1}\right\}$. The map $\left\{a_{i} \mapsto W_{i}, a_{i}^{-1} \mapsto\left(W_{i}\right)^{-1} \mid 1 \leq i \leq m\right\}$ induces an endomorphism $\tau: M \rightarrow M$. In this notation the label of $\partial D_{n}$ beginning at the basepoint of $D_{n}$ is $\tau^{n}(R) \in M$.

For each $n$ we orient $D_{n}$ and lift it to $\hat{D}_{n} \subset \hat{X}$, thus obtaining in an obvious manner an element $d_{n} \in C_{2}(\hat{X})$ of the 2-chain-complex of $\hat{X}$.

Since the boundary of each $D_{n}$ diagram is mapped to $B \subset X$, it is true that the boundary of each $\hat{D}_{n}$ is mapped to $\hat{B}$. Therefore for each $n$ we have

$$
\partial d_{n} \in C_{1}(\hat{B}) \subset C_{1}(\hat{X}) .
$$

Observe that the image of $t \in G$ has infinite order in the natural homomorphism $G \rightarrow G /\langle\langle A\rangle\rangle \cong Z$. It follows that for $n \neq 1, t^{n} \notin A$, so the paths in $\hat{X}$ beginning at the basepoint and labelled by $t^{n}$ all have distinct endpoints.

Note that the $r$-labelled 2-cell at the center of each disc-diagram $D_{n}$ is connected to the basepoint of $D_{n}$ by a path labeled by $t^{n}$.

It follows that the $r$-labelled 2-cells at the centers of distinct $D_{n}$ diagrams lift to distinct $r$-labelled 2-cells in $\hat{X}$.

It is then easy to see that the set of 2-chains $\left\{d_{n} \mid n \geq 0\right\} \subset C_{2}(\hat{X})$ forms a basis for the submodule that it generates.

It follows from equation (2) that $\partial$ maps this infinite rank submodule of $C_{2}(\hat{X})$ to the finite rank (actually rank $m$ ) submodule $C_{1}(\hat{B}) \subset C_{1}(\hat{X})$. The kernel of 
$\partial$ restricted to this infinite rank submodule must be of infinite rank. Therefore $H_{2}(\hat{X})$ must be of infinite rank as well.

2.2 Example: An incoherent small-cancellation group. It is easy to construct examples of presentations with the form of Theorem 2.1 which are smallcancellation presentations (without torsion), and therefore aspherical [9]. For instance,

$$
\left\langle a, b, t \mid a^{1} b^{1} a^{2} b^{2} \cdots a^{30} b^{30}=1, a^{t}=a b^{10} a b^{11} \ldots a b^{30}, b^{t}=b a^{10} b a^{11} \ldots b a^{30}\right\rangle .
$$

\subsection{Example: An incoherent 2-relator group.}

$$
\left\langle a, b, t \mid a^{t}=a^{10} b a^{10}, b^{t}=b^{10} a b^{10}, a^{9} b^{2} a^{8} b^{3} a^{7} b^{4} a^{6} b^{5} a^{5} b^{6} a^{4} b^{7} a^{3} b^{8} a^{2} b^{9}=1\right\rangle .
$$

The presentation above satisfies the $C(6)$ small cancellation condition and so is aspherical. A 2-relator presentation may be obtained by using the substitution $b=a^{-10} a^{t} a^{-10}$, followed by a Tietze transformation eliminating the generator $b$ and the relator $a^{t}=a^{10} b a^{10}$. Substantially less complicated incoherent 2-relator examples could be formed using the same idea, but proving that the presentation in the first step is aspherical is more difficult.

2.4 Example: Incoherent negatively curved. Here is an example of a negatively curved 2-complex which is of the desired form. Metrize each 2-cell of the standard complex of

$$
\left.\left\langle a_{1}, \ldots, a_{7}, t\right| a_{7} a_{6} a_{5} a_{4} a_{3} a_{2} a_{1}, a_{i}^{t}=a_{i} a_{i} a_{i+1} a_{i+3}(\text { coefficients } \bmod 7)\right\rangle
$$

as a regular hyperbolic heptagon with angles $\frac{2 \pi}{3}$.

One must only check that the presentation has no pieces of length 2 in the sense of Lyndon (see [9]). This means that no subword of length 2 appears more than once among the cyclic conjugates of the relators and their inverses.

\section{ACKNowledgement}

It is a pleasure to thank my advisor M. R. Bridson and S. M. Gersten for helpful comments and encouragement. I am grateful as well to the referees that greatly improved the exposition of this paper.

\section{REFERENCES}

[1] G. Baumslag, Some Problems in One-Relator Groups, in: Proc. Conf. Canberra 1973, Lecture notes in Math Vol. 372 (1973), 75-81. MR 51:717

[2] G. Baumslag, M. R. Bridson, C. F. Miller III, H. Short, Subgroups of Automatic Groups, pre-print.

[3] G. Baumslag, C. F. Miller III, H. Short, Unsolvable problems about Small Cancellation and Word Hyperbolic Groups, Bull. London Math. Soc. 26 (1994), 97-101. MR 94i:20053

[4] G. Baumslag and J. E. Roseblade, Subgroups of Direct Product of Two Free Groups, J. London Math. Soc. (2) 30 (1984), 44-52. MR 86d:20028

[5] R. Bieri, Homological dimension of discrete groups; Queen Mary College Math. Notes, Queen Mary College, London, 1976. MR 57:6224

[6] M. R. Bridson and A. Haefliger, Metric spaces of nonpositive curvature, to appear.

[7] S. M. Gersten, Questions on Geometric Group Theory for the Max Dehn Seminar, available by ftp at: ftp.math.utah.edu /u/ma/gersten/MaxDehnSeminar (1995).

[8] M. Gromov, Hyperbolic Groups, in: Essays in Group Theory (S.M. Gersten ed.) (1987). MR 89e:20070

[9] R. C. Lyndon, and P. E. Schupp, Combinatorial Group Theory, Springer-Verlag, 1977. MR 58:28182 
[10] E. Rips, Subgroups of Small Cancellation Groups, Bull. London Math. Soc. (1982), 45-47. MR 83c: 20049

[11] G. P. Scott, Finitely generated 3-manifold groups are finitely presented, J. London Math. Soc. 6 (1973), 437-440. MR 52:1660

[12] J. P. Serre, Problems, in: Proc. Conf. Canberra 1973, Lecture notes in Math Vol. 372 (1974), 734-735. MR 51:656

Department of Mathematics, Fine Hall, Princeton University, Princeton, New JerSEY 08544

Current address: Department of Mathematics, White Hall, Cornell University, Ithaca, New York 14853

E-mail address: daniwise@math.cornell.edu 